\title{
The History and Current Situation of the Development of Urban Management Major in China
}

\author{
Ya-juan Sun \\ Xijing University, Xi'an, Shaanxi, China \\ 723240327@qq.com
}

Keywords: City Management Major, Historical Evolution, Current Situation

\begin{abstract}
The development of urban management specialty can be divided into three stages: germination, start and rapid development; the distribution of professional geographical distribution is not balanced; the colleges and universities carry out professional construction according to their own subject advantages and teachers' advantages.
\end{abstract}

\section{Introduction}

For the first time, the major of urban management has been included in the catalogue of the undergraduate course of colleges and universities in the 2012 edition, which reflects the scientific judgment of the state for the future needs of urban management professionals. As a rapid urbanization background, urban management specialty is rising rapidly in order to adapt to the specialization of urban management and the demand for special talents in urban management.

\section{The development course of urban management specialty}

The undergraduate training of urban management has been cultivated for more than 30 years in China, and its development process is divided into three stages, namely, the germination stage, the initial stage and the rapid development stage ${ }^{[1]}$.

\subsection{Germination stage (before 2000)}

From 1984 to 2000, it was the embryonic stage of urban management. At this stage, there is only one university -- Capital University of Economics and Business has established a major in urban management. In 1984, Capital University of Economics and Business set up the direction of urban management in economics. In 1985, with the approval of the former National Education Commission, the city management specialty was formally established and began to recruit students. In 1993, the major of urban management in Capital University of Economics and Business stopped enrollment. Until 2000, Chinese universities did not recruit urban management undergraduates.

\subsection{Start stage (2001 -2011)}

From 2001 to 2011, it is the initial stage of urban management. Since 2001, the major of urban management as an undergraduate specialty in the Ministry of education has been self-designed. As shown in Figure 1, 19 universities, such as Peking University, Renmin University of China, Yunnan University, East China University of Science and Technology, have set up urban management major successively. 


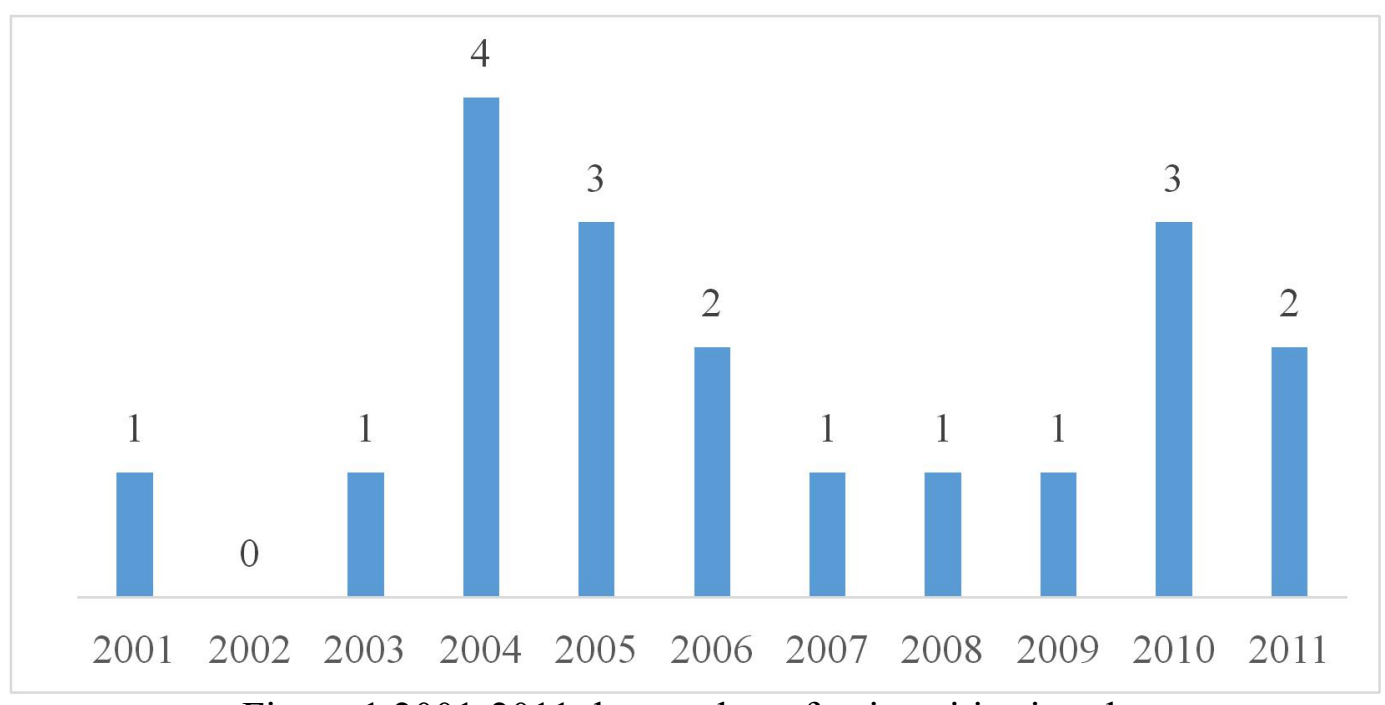

Figure $12001-2011$ the number of universities in urban management

\subsection{Rapid development phase (2012 to present)}

Since 2012, it is a fast developing stage of urban management. In 2012, the Ministry of education incorporated the specialty of urban management into the catalogue, and many universities began to apply for the major of urban management. As shown in Figure 2, from 2012 to 2017, a total of 47 colleges and universities offered urban management.

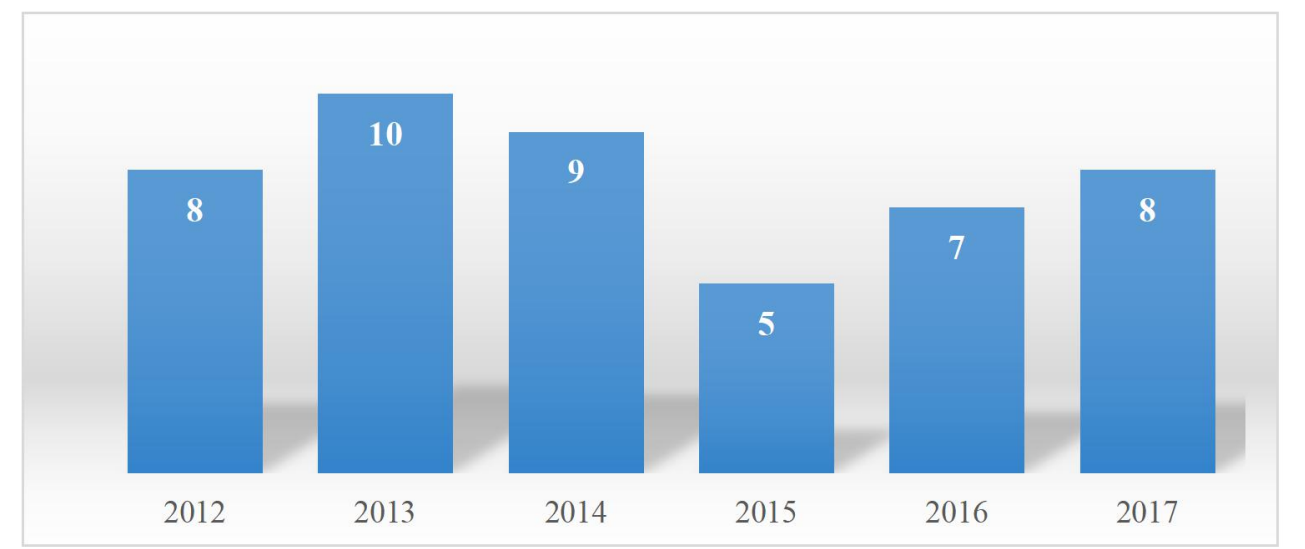

Figure 2 2002-2017 the number of universities in urban management

\section{Regional distribution of urban management specialty}

According to statistics, a total of 66 universities in China are now offering urban management. As shown in Figure 3, the largest number of universities in eastern China has a major in urban management, accounting for 17 , accounting for $28 \%$ of the country's urban management specialty, followed by 13 in the southwest, 21\%, 11 and 18\% in North China; fourth in Central China, 7, 12\% and fifth, Southern China and the northwest, fifth, and the northwest region, and the fifth, and the northwest region, and the northwest region, and the fifth. There are 5, accounting for $8 \%$, sixth in the northeast, and 3 in $5 \%$. 




Figure 3 regional distribution of urban management specialty

From the provinces and cities, the city with the most major city management specialties is Beijing, with 6 in all; second in Shandong and Zhejiang provinces, 5 universities each have urban management specialties; third are Yunnan Province, Guizhou province and Guangxi Province, each has 4; the fourth is Chongqing, Shaanxi province and Henan Province, each has 3; fifth is Tianjin city. There are 2 in Fujian, Jiangsu, Sichuan, Hubei, Hunan, Jilin and Shanxi, and sixth are Shanghai, Jiangxi, Guangdong, Liaoning, Gansu, Anhui, and 1.

\section{The present situation of the construction of urban management specialty \\ 4.1 Culture Target}

The economic management colleges, such as Capital University of Economics and Business, Renmin University of China, and other economic management colleges and universities focus on the cultivation of urban management talents with distinctive features of applied and compound economic management specialty. Zhejiang agroforestry university is supported by law, and is committed to cultivating professional talents in administrative law enforcement of urban management; the Shenyang architecture of architecture colleges and Universities The universities, the Jilin Architecture University and the Hunan City University are relying on their own civil construction and urban planning and other dominant disciplines, focusing on the training of urban management complex and applied talents with civil construction background, and the detailed management of municipal engineering, transportation planning and landscape architecture involving urban management. The project is set up as a specific speciality for more targeted training.

\subsection{Culture Direction}

Colleges and universities mainly aim at the training direction of urban management major according to their own conditions and characteristics ${ }^{[2]}$, such as the Zhejiang Gongshang University mainly from the perspective of urban planning and construction management, the Yunnan University emphasizes the combination of science and technology management, focusing on the students in urban planning, city management, modern information management technology, and so on. The basic 
training of the application of the subject; the two directions of the urban community management, the construction and management of the small cities, and the Shandong Institute of Business and Technology as the application and compound talents for the cultivation of municipal administration, community management and property management, and the Tianjin University to locate the livable city, the digital city and the urban safety. And several aspects of emergency management.

\subsection{Curriculum System}

The curriculum system of urban management major in various universities is basically the same. It can be summed up in the following four major course systems: basic general course, major core curriculum, characteristic elective course and practical skill course ${ }^{[3]}$.Although the curriculum system of urban management in various colleges and universities is as much as possible to highlight the professional characteristics, there are three common problems in the actual operation. One is the discontinuity of the curriculum system, the relative isolation of the curriculum, and the inability to link up; the two is the repetition of the contents of some courses; and the three is the lack of teaching materials in the partial curriculum.

\subsection{Practice Link}

It is an urgent task for the construction of urban management to train the applied compound talents with innovative spirit and practical ability ${ }^{[4]}$. All schools have set up experimental training courses for this purpose, and have a certain number of practical teaching. Generally speaking, the experimental training of core courses of urban management needs to be broken through. In addition, the major urban management major is to make use of the practical teaching base of other specialties. There is no connection between the urban comprehensive law enforcement bureau, the Construction Bureau, the Municipal Bureau, the urban community and other departments which are highly related to the city management specialty. The number of existing bases in the admission of students is limited and the centralized practice is in the form of form. Relying on students to decentralize their internships, the quality of internship can not be controlled, and the practice base of urban management specialty needs to be further developed.

\subsection{Direction of Employment}

In all colleges and universities, in addition to a few well-known research universities, there is a common problem of low rate of first volunteer filling and high rate of conversion of professional rate $^{[5]}$. In addition, in view of the limitations of unit identity and compiling (which must be admitted), recruiting major and the number of recruits, there are only a few posts for urban management students, although the city management of colleges and Universities The employment data of science and technology are quite good, but there are also some internal risks. More than half of the students are engaged in non city management after graduation.

\section{References}

[1] Liu Guangzhu. History and current situation of urban management specialty in Chinese universities [J]. urban management and technology, 2016,18 (05): 11-12.

[2] He Jixin, Liu Yanping. Exploration and Reflection on the training of professionals in urban management [J]. urban management and technology, 2016,18 (05): 21-23.

[3] Wang Qi. A comparative study on the training mode of urban management professionals in Chinese colleges and Universities -- a text analysis based on talent training program [J]. Journal of Hunan University of Commerce, 2014,21 (03): 113-117.

[4] Liu Guangzhu. Build a cross-strait exchange platform to promote the cooperation and development of urban management -- Book Review on urban management papers on both sides of the Straits, [J]. city management and science and technology, 2018,20 (02): 95-96.

[5] Zhang Yuwen, Zou Yonghua. Enlightenment from the training mode of urban professionals in American universities [J]. urban management and technology, 2016,18 (05): 19-21. 